\title{
Spin-orbit-coupled topological Fulde-Ferrell states of fermions in a harmonic trap
}

\author{
Lei Jiang, ${ }^{1}$ Eite Tiesinga, ${ }^{1}$ Xia-Ji Liu, ${ }^{2} \mathrm{Hui} \mathrm{Hu},{ }^{2}$ and $\mathrm{Han} \mathrm{Pu}^{3}$ \\ ${ }^{1}$ Joint Quantum Institute, University of Maryland, and National Institute of Standards and Technology, Gaithersburg, Maryland 20899, USA \\ ${ }^{2}$ Centre for Atom Optics and Ultrafast Spectroscopy, Swinburne University of Technology, Melbourne 3122, Australia \\ ${ }^{3}$ Department of Physics and Astronomy, and Rice Quantum Institute, Rice University, Houston, Texas 77251, USA
}

(Received 24 April 2014; published 7 November 2014)

\begin{abstract}
Motivated by recent experimental breakthroughs in generating spin-orbit coupling in ultracold Fermi gases using Raman laser beams, we present a systematic study of spin-orbit-coupled Fermi gases confined in a quasione-dimensional trap in the presence of an in-plane Zeeman field (which can be realized using a finite two-photon Raman detuning). We find that a topological Fulde-Ferrell state will emerge, featuring finite-momentum Cooper pairing and zero-energy Majorana excitations localized near the edge of the trap based on the self-consistent Bogoliubov-de Gennes (BdG) equations. We find analytically the wave functions of the Majorana modes. Finally, using the time-dependent BdG, we show how the finite-momentum pairing field manifests itself in the expansion dynamics of the atomic cloud.
\end{abstract}

DOI: 10.1103/PhysRevA.90.053606

PACS number(s): 67.85.Lm, 03.65.Vf, 03.75.Ss, 05.30.Fk

\section{INTRODUCTION}

Over the past few years, spin-orbit-coupled quantum gases have received a great amount of interest in both the cold atom and condensed-matter communities [1-12]. This can be largely attributed to the fact that such a system can potentially realize exotic quantum phases in a controlled fashion. So far only one type of spin-orbit coupling (SOC) - the equal-weight Rashba and Dresselhaus SOC—has been realized [13,14], although several theoretical schemes have been proposed to realize more general types of SOC. Nevertheless, the experimentally realized SOC has already been shown to give rise to several interesting quantum phases. These include the topological superfluid phase in a one-dimensional (1D) system supporting Majorana modes near the boundaries [15-18], and the Fulde-Ferrell (FF) superfluid state featuring finitemomentum Cooper pairing [19-25]. Furthermore, in a 1D setting, these two features can coexist where one realizes an exotic topological FF superfluid [26-29]. Early experiments explored the possibility of the Fulde-Ferrell-Larkin-Ovchinnikov state in spin-imbalanced cold atoms [30-32], with indirect evidence only coming from a quasi-1D setup [33].

Previous work on topological FF phases focused on homogeneous $1 \mathrm{D}$ gases. In this work, we consider a system in a realistic quasi-1D harmonic trapping potential and investigate how signatures of exotic phases can be probed in practice. Such a quasi-1D system can be realized by confining atoms in strong two-dimensional (2D) optical lattices [33]. The presence of a trapping potential suppresses quantum fluctuations and makes mean-field calculations qualitatively reliable [34,35]. The two goals of this work are as follows: (1) We show how a Majorana mode, the smoking gun of the topological order, manifests itself in the density of states (DOS) in both real and momentum spaces. We obtain the wave functions of the Majorana quasiparticle states analytically and show that they are in good agreement with numerical results based on the Bogoliubov-de Gennes (BdG) formalism. (2) We show how finite-momentum Cooper pairing, the telltale signal of the FF phase, leaves detectable traces in the expansion dynamics of the atomic cloud.
The paper is organized as follows. In Sec. II, we introduce our model Hamiltonian. The self-consistence BdG formalism - both the time-independent and the timedependent versions-is discussed in Sec. III. In Sec. IV, we apply the time-independent $\mathrm{BdG}$ equation to obtain the ground state of the system and, in particular, we calculate the wave function of the Majorana mode in the topological regime, and its effect on the DOS in both real and momentum spaces. The numerically calculated wave function is then compared with the result obtained from an analytic method and good agreement is found. In Sec. V, the time-dependent BdG equation is used to investigate the expansion dynamics of the FF superfluid. Finally, concluding remarks are contained in Sec. VI.

\section{MODEL}

We consider a spin-1/2 Fermi gas confined in a $1 \mathrm{D}$ harmonic trap. Its Hamiltonian is given by $H=H_{0}+H_{\text {int }}$, with single-particle component $H_{0}$ and interacting component $H_{\text {int }}$ describing the $s$-wave contact interaction. In fact,

$$
\begin{aligned}
H_{0}= & \int d x \psi_{\uparrow}^{\dagger}(x)\left[H_{s}+\delta / 2\right] \psi_{\uparrow}(x) \\
& +\int d x \psi_{\downarrow}^{\dagger}(x)\left[H_{s}-\delta / 2\right] \psi_{\downarrow}(x) \\
& -\frac{\Omega_{R}}{2} \int d x\left[\psi_{\uparrow}^{\dagger}(x) e^{i 2 k_{R} x} \psi_{\downarrow}(x)+\text { H.c. }\right], \\
H_{\text {int }}= & g_{1 \mathrm{D}} \int d x \psi_{\uparrow}^{\dagger}(x) \psi_{\downarrow}^{\dagger}(x) \psi_{\downarrow}(x) \psi_{\uparrow}(x),
\end{aligned}
$$

where $\psi_{\uparrow}(x), \psi_{\downarrow}(x)$ are Fermi annihilation operators for the two spin states. The Hamiltonian $H_{s}=-\frac{\hbar^{2}}{2 m} \frac{\partial^{2}}{\partial x^{2}}-\mu+V_{T}(x)$, with $\mu$ being the chemical potential and $V_{T}(x)=\frac{1}{2} m \omega^{2} x^{2}$ the harmonic trapping potential with a frequency $\omega$. The constants $\delta$ and $\Omega_{R}$ represent the detuning and strength of the twophoton Raman coupling, respectively, $2 \hbar k_{R}$ is the photon recoil momentum imparted to the atoms from the Raman lasers, and $\hbar$ is the reduced Planck constant. Finally, $g_{1 \mathrm{D}}$ is the 1D two-body $s$-wave interaction strength. 
After applying the local gauge transformation

$$
\begin{aligned}
& \psi_{\uparrow}(x)=e^{i k_{R} x}\left[\phi_{\uparrow}(x)-i \phi_{\downarrow}(x)\right] / \sqrt{2}, \\
& \psi_{\downarrow}(x)=e^{-i k_{R} x}\left[\phi_{\uparrow}(x)+i \phi_{\downarrow}(x)\right] / \sqrt{2},
\end{aligned}
$$

the single-particle Hamiltonian $H_{0}$ becomes

$$
H_{0}=\int d x \phi^{\dagger}\left[H_{s}+\left(-i \lambda \partial_{x}+v\right) \sigma_{y}-h \sigma_{z}\right] \phi,
$$

where $\phi=\left[\phi_{\uparrow}(x), \phi_{\downarrow}(x)\right]^{T}$ and we have dropped a constant corresponding to the atomic recoil energy. In writing Eq. (1), we have defined the spin-orbit-coupling constant $\lambda \equiv \hbar^{2} k_{R} / m$, the effective out-of-plane Zeeman field $h \equiv \Omega_{R} / 2$, and the effective in-plane Zeeman field $v \equiv \delta / 2$. It is convenient to define the effective Zeeman field strength $b \equiv \sqrt{h^{2}+v^{2}}$. The operators $\sigma_{y}$ and $\sigma_{z}$ are Pauli matrices in the atomic spin basis. The interaction Hamiltonian $H_{\text {int }}$ is invariant under this gauge transformation.

\section{BOGOLIUBOV-DE GENNES FORMALISM}

In the mean-field $\mathrm{BdG}$ approximation, we assume a nonzero complex order parameter or gap

$$
\Delta(x) \equiv-g_{1 \mathrm{D}}\left\langle\phi_{\downarrow}(x) \phi_{\uparrow}(x)\right\rangle=-i g_{1 \mathrm{D}}\left\langle\psi_{\downarrow}(x) \psi_{\uparrow}(x)\right\rangle,
$$

and in terms of the Nambu spinor $\Phi(x)=$ $\left[\phi_{\uparrow}(x), \phi_{\downarrow}(x), \phi_{\uparrow}^{+}(x), \phi_{\downarrow}^{+}(x)\right]^{T}$ the mean-field Hamiltonian becomes

$$
H_{\mathrm{mf}}=\frac{1}{2} \int d x \Phi^{\dagger}(x) H_{\mathrm{BdG}} \Phi(x)+\operatorname{Tr}\left[H_{s}\right]-\int d x \frac{|\Delta(x)|^{2}}{g_{1 \mathrm{D}}},
$$

where

$$
H_{\mathrm{BdG}}=\left[\begin{array}{cccc}
H_{s}-h & -\lambda \partial_{x}-i \nu & 0 & -\Delta(x) \\
\lambda \partial_{x}+i v & H_{s}+h & \Delta(x) & 0 \\
0 & \Delta^{*}(x) & -H_{s}+h & \lambda \partial_{x}-i v \\
-\Delta^{*}(x) & 0 & -\lambda \partial_{x}+i v & -H_{s}-h
\end{array}\right] .
$$
ing

The Bogoliubov quasiparticles are obtained by diagonaliz-

$$
H_{\mathrm{BdG}} \varphi_{\eta}(x)=E_{\eta} \varphi_{\eta}(x)
$$

with energies $E_{\eta}$ and wave functions $\varphi_{\eta}(x)=$ $\left[u_{\uparrow \eta}(x), u_{\downarrow \eta}(x), v_{\uparrow \eta}(x), v_{\downarrow \eta}(x)\right]^{T} \quad$ indexed $\quad$ by subscript $\eta=1,2,3 \ldots$ The wave functions are normalized such that $\sum_{\sigma=\uparrow, \downarrow} \int d x\left(\left|u_{\sigma \eta}(x)\right|^{2}+\left|v_{\sigma \eta}(x)\right|^{2}\right)=1$. The order parameter

$$
\Delta(x)=-\frac{g_{1 \mathrm{D}}}{2} \sum_{\eta}\left[u_{\uparrow \eta} v_{\downarrow \eta}^{*} f\left(E_{\eta}\right)+u_{\downarrow \eta} v_{\uparrow \eta}^{*} f\left(-E_{\eta}\right)\right]
$$

must be solved self-consistently, where $f(E)$ is the FermiDirac distribution function $f(E)=1 /\left[e^{E / k_{B} T}+1\right]$ and $T$ is the temperature. Here we present results for $T=0$.

To solve the eigenvalue problem, we use the discrete variable representation of the plane-wave basis [36]. We employ 1001 plane-wave bases and the total number of atoms is $N=60$. In the harmonic trap with frequency $\omega$, we define the noninteracting Fermi energy $E_{F}=\hbar \omega N / 2$, the Fermi wave number $k_{F}$, obtained from $E_{F}=\hbar^{2} k_{F}^{2} /(2 m)$, and the Thomas-Fermi radius $x_{\mathrm{TF}}=\sqrt{N \hbar /(m \omega)}$. Throughout we use $E_{F}$ and $x_{\mathrm{TF}}$ as the natural energy and length scale, respectively. Equation (2) is solved by using a "hybrid" method of Refs. [35,37-39]. We start with an initial guess of the order parameter. We then find all the eigenpairs of $H_{\mathrm{BdG}}$ with energy $|E| \leq E_{c}$, where $E_{c}$ is a cutoff energy that is chosen to be large compared to the Fermi energy but small compared to the full spectral width of the discretized $H_{\mathrm{BdG}}$. Typically we take $E_{c}=8 E_{F}$. For states above the energy cutoff, we employ a semiclassical method based on the local density approximation. The updated order parameter is calculated by combining the contributions from the numerical and semiclassical solutions. The procedure is repeated until convergence of the order parameter is reached.

It is convenient to characterize the interaction strength $g_{1 \mathrm{D}}$ by a dimensionless interaction parameter $\gamma \equiv-m g_{1 \mathrm{D}} /\left(\hbar^{2} n_{0}\right)$ which represents the ratio between the interaction and kinetic energy. Here $n_{0}=(2 / \pi) \sqrt{N m \omega / \hbar}$ is the total atomic density at the trap center in the Thomas-Fermi approximation.

To study expansion dynamics, we solve the time-dependent BdG equation [40-43]

$$
i \hbar \partial_{t} \varphi_{\eta}(x, t)=H_{\mathrm{BdG}} \varphi_{\eta}(x, t),
$$

with the initial wave function of $\varphi_{\eta}(x, 0)$ set by the ground state of the trapped system obtained from Eq. (2). We use the Runge-Kutta method for the time propagation. The kinetic energy and the spin-orbit-coupling term are propagated in the interaction picture using a fast Fourier transform.

\section{TOPOLOGICAL FF STATE AND MAJORANA MODES}

In Fig. 1, we characterize the properties of the topological FF state in the trapped system using experimentally relevant parameters. Figure 1(a) shows atom density profiles of both spin species, $n_{\sigma}(x)=\left\langle\psi_{\sigma}^{\dagger}(x) \psi_{\sigma}(x)\right\rangle$, along with the magnitude of the order parameter $|\Delta(x)|$. The densities peak at the center of the trap, while $|\Delta(x)|$ has a minimum at the center and reaches its maximum value near the edge of the cloud. This is due to a peculiar property of 1D quantum gases [35]: For sufficiently large density, the effect of the interaction and thus the order parameter is enhanced by reducing the number density, while near the edge of the cloud, the order parameter has a power-law dependence on density.

For positive two-photon detuning $\delta=2 v$ used in the calculation, the effective chemical potential for spin-up atoms, $\mu-\delta / 2$, is lower than that for spin-down atoms, $\mu+\delta / 2$. Consequently, spin-up (spin-down) represents the minority (majority) species. As we change the sign of $\delta$, the density profiles of the two spin species switch. This follows from a symmetry of the BdG Hamiltonian for this system: Under the simultaneous transformation $v \rightarrow-v, \Delta(x) \rightarrow-\Delta(-x)$ and

$$
\begin{aligned}
& {\left[u_{\uparrow \eta}(x), u_{\downarrow \eta}(x), v_{\uparrow \eta}(x), v_{\downarrow \eta}(x)\right]} \\
& \quad \rightarrow\left[u_{\uparrow \eta}(-x),-u_{\downarrow \eta}(-x), v_{\uparrow \eta}(-x),-v_{\downarrow \eta}(-x)\right] .
\end{aligned}
$$

\section{Equation (2) remains invariant.}

Figure 1(b) shows the real and imaginary parts of the order parameter. Near the center of the trap, the order parameter has the plane-wave form $\Delta(x) \simeq \Delta_{0} e^{i q x}$, indicating finitemomentum pairing. This is the characteristic signature of a FF superfluid state $[44,45]$. The pairing momentum $q$ can be 

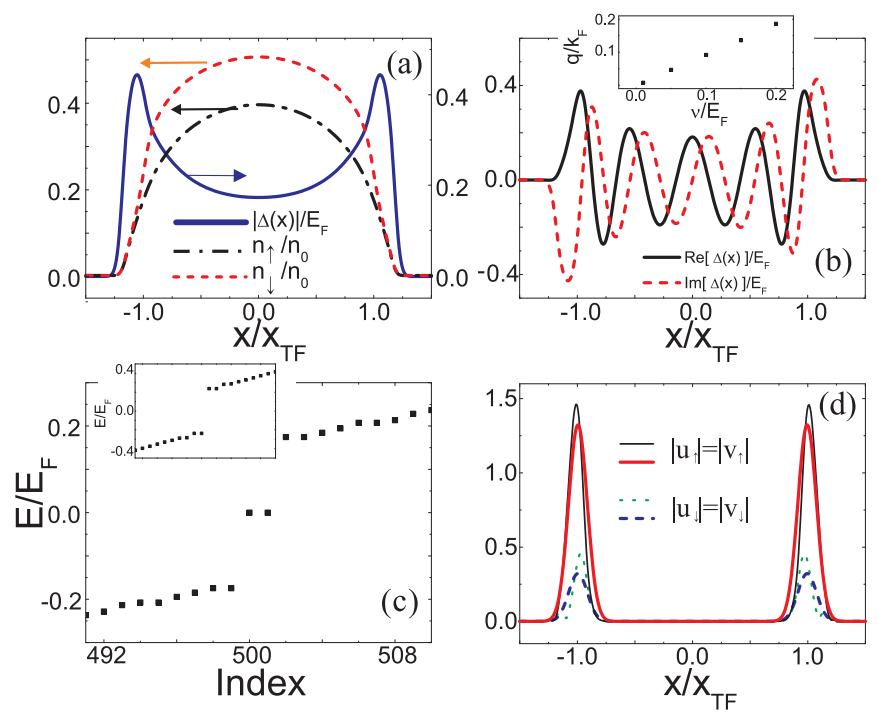

FIG. 1. (Color online) (a) Density profiles $n_{\sigma}(x)$ in units of $n_{0}$ (left $y$ axis) and order parameter profile $|\Delta(x)|$ in units of $E_{F}$ (right $y$ axis). (b) The real and imaginary parts of the order parameter profile. The inset shows the pairing momentum $q$ as a function of $v$. (c) Low-lying spectrum of quasiparticle excitation. The inset shows the corresponding spectrum of a topologically trivial phase with $v=0$ and $h=0.5 E_{F}$ with other parameters the same as those of the rest of the figure. (d) Wave function of the Majorana modes. The thin (thick) lines represent numerical (analytical) results. The parameters used for this figure are $\gamma=2.2, \lambda=1.5 E_{F} / k_{F}, h=0.8 E_{F}$, and $v=0.2 E_{F}$ unless otherwise noted.

easily extracted from the data. The inset of Fig. 1(b) shows that $q$ increases nearly linearly as a function of $v$.

In Fig. 1(c), we show the low-lying quasiparticle excitation spectrum of the system. The two dots with zero energy are the Majorana modes characterizing the nontrivial topological nature of the system. The Majorana modes exist inside the gap in the energy spectrum. For comparison, a spectrum for a topologically trivial system is shown in the inset, where no states inside the gap exist. The wave functions of the two Majorana modes are plotted in Fig. 1(d). The two Majorana modes are spatially localized near the two edges of the cloud at $x= \pm x_{\mathrm{TF}}$. Their wave functions satisfy the condition $\left|u_{\sigma}\right|=$ $\left|v_{\sigma}\right|$. The contribution of the Majorana modes to the order parameter is negligible. This is not surprising as many states contribute to $\Delta(x)$.

Figure 2 shows the density of states both in real space and momentum space and defined by

$$
\begin{aligned}
& \rho(x, \omega)=\frac{1}{2} \sum_{\sigma \eta}\left[\left|u_{\sigma \eta}\right|^{2} \delta\left(\omega-E_{\eta}\right)+\left|v_{\sigma \eta}\right|^{2} \delta\left(\omega+E_{\eta}\right)\right], \\
& \tilde{\rho}(k, \omega)=\frac{1}{2} \sum_{\sigma \eta}\left[\left|\tilde{u}_{\sigma \eta}\right|^{2} \delta\left(\omega-E_{\eta}\right)+\left|\tilde{v}_{\sigma \eta}\right|^{2} \delta\left(\omega+E_{\eta}\right)\right],
\end{aligned}
$$

where $\tilde{u}_{\sigma \eta}(k)=\int u_{\sigma \eta}(x) e^{i k x} d x$ and $\tilde{v}_{\sigma \eta}(k)=\int v_{\sigma \eta}(x) e^{i k x} d x$ are the Fourier transforms of $u_{\sigma \eta}(x)$ and $v_{\sigma \eta}(x)$, respectively. In the calculations, the Dirac $\delta$ function is replaced by a Gaussian with a width on the order of spacings in the energy spectrum away from the gap. The zero-energy Majorana modes in the plots of the density of states are easily identified. They
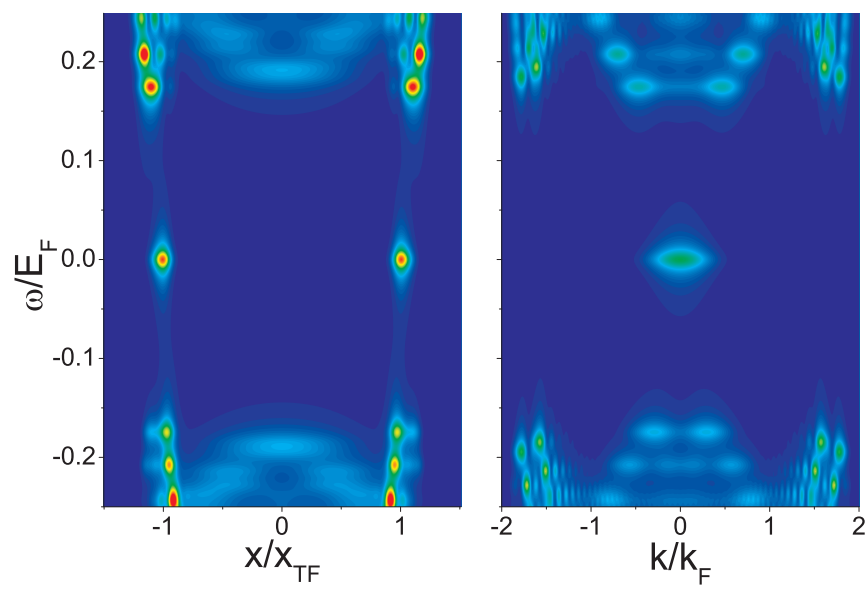

FIG. 2. (Color online) Density of states in real space (left panel) and in momentum space (right panel). The parameters are the same as in Fig. 1. The brighter color represents a higher density of states.

are localized in both real space (near $\pm x_{\mathrm{TF}}$ ) and momentum space (around $k=0$ ). In principle, the density of states can be measured in experiment by using spatial- and momentumresolved radio-frequency spectroscopy [46].

Analytical results for Majorana wave functions. We can derive analytic formulas for the Majorana wave functions. Our method relies on a linearization of the Bogoliubov-de Gennes (BdG) Hamiltonian based on the fact that Majorana modes are localized in both real (near the trap edges) and momentum (near zero momentum) spaces. To this end, we divide the BdG Hamiltonian $H_{\mathrm{BdG}}=H_{0}+H_{1}+H_{2}$, where $H_{0}$, a local Hamiltonian at real-space point $x_{0}$ near which the Majorana modes are localized, is given by

$$
H_{0}=\left(\begin{array}{cc}
V_{0} I-h \sigma_{z}+v \sigma_{y} & -i \Delta_{0} \sigma_{y} \\
i \Delta_{0}^{*} \sigma_{y} & -V_{0} I+h \sigma_{z}+v \sigma_{y}
\end{array}\right),
$$

where $V_{0}=\frac{1}{2} m \omega^{2} x_{0}^{2}-\mu$ and $I$ is the $2 \times 2$ identity matrix.

$H_{1}$ contains the linearized potential and spin-orbit-coupling term given by

$$
H_{1}=\left(\begin{array}{cc}
V_{1}(x) I+\lambda \hat{k} \sigma_{y} & 0 \\
0 & -V_{1}(x) I-\lambda \hat{k} \sigma_{y}
\end{array}\right),
$$

where $V_{1}(x)=m \omega^{2} x_{0}\left(x-x_{0}\right)$. Finally, $H_{2}$ contains the kinetic energy and corrections of the order parameter $\Delta(x)$ near $\Delta_{0} \equiv \Delta\left(x_{0}\right)$. We neglect $H_{2}$ in our calculation as its effect is small.

The local Hamiltonian $H_{0}$ has two degenerate zero-energy eigenstates when

$$
b=\sqrt{\left(\frac{1}{2} m \omega^{2} x_{0}^{2}-\mu\right)^{2}+\left|\Delta_{0}\right|^{2}},
$$

where the effective Zeeman field strength $b=\sqrt{h^{2}+v^{2}}$ combines both the in- and out-of-plane fields. This equation sets the value of $x_{0}$. In our parameter region, $x_{0}$ has two solutions $\pm\left|x_{0}\right|$, localized near the left and right edge of the harmonic trap, respectively. The corresponding zero-energy 
eigenstates are

$$
\begin{aligned}
& |1\rangle=\left(-\frac{a+h}{\sqrt{2 b^{2}+2 a h}}, \frac{i v}{\sqrt{2 b^{2}+2 a h}}, 0, \frac{\Delta_{0}^{*}}{\sqrt{2 b^{2}+2 a h}}\right)^{T}, \\
& |2\rangle=\left(\frac{i v}{\sqrt{2 b^{2}-2 a h}}, \frac{a-h}{\sqrt{2 b^{2}-2 a h}}, \frac{\Delta_{0}^{*}}{\sqrt{2 b^{2}-2 a h}}, 0\right)^{T},
\end{aligned}
$$

where $a=\sqrt{b^{2}-\left|\Delta_{0}\right|^{2}}$. The two other eigenstates of $H_{0}$ have finite energies $\pm 2 b$. As we are only interested in zero-energy states of the Hamiltonian, we neglect the effects of these two energy states in the following.

Next, we treat $H_{1}$ as a small perturbation to $H_{0}$ in the subspace spanned by $\{|1\rangle,|2\rangle\}$. In this two-dimensional subspace, $H_{1}$ takes the form

$$
H_{1}^{\mathrm{eff}}=\left(\begin{array}{cc}
\frac{a+h}{b^{2}+a h}(a \tau \hat{x}-\nu \lambda \hat{k}), & \frac{a v \tau \hat{x}-\left(v^{2}-\left|\Delta_{0}\right|^{2}\right) \lambda \hat{k}}{i \sqrt{b^{4}-a^{2} h^{2}}} \\
\frac{a \nu \tau \hat{x}-\left(v^{2}-\left|\Delta_{0}\right|^{2}\right) \lambda \hat{k}}{-i \sqrt{b^{4}-a^{2} h^{2}}}, & \frac{a-h}{b^{2}-a h}(a \tau \hat{x}-\nu \lambda \hat{k})
\end{array}\right),
$$

where $\tau=m \omega^{2} x_{0}$ and $\hat{x}=x-x_{0}$.
In real space, the momentum operator takes the form $\hat{k}=$ $-i d / d x$ and we search for a zero-energy eigenstate $(p, q)^{T}$ of $H_{1}^{\text {eff }}$. This leads to coupled differential equations for $p$ and $q$ with unit-normalized Gaussian solutions

$$
\begin{aligned}
& p=\frac{1}{\sqrt[4]{\pi \sigma^{2}}} e^{-\frac{\left(x-x_{0}\right)^{2}}{2 \sigma^{2}}}, \\
& q=-\operatorname{sgn}(\tau) \sqrt{\frac{h+a}{h-a}} \sqrt{\frac{b^{2}-a h}{b^{2}+a h}} p,
\end{aligned}
$$

where $\operatorname{sgn}(z)$ is the sign of $z$ and the width $\sigma$ is determined by $\sigma^{2}=\lambda \sqrt{h^{2}-a^{2}} /(|\tau| a)$. The two corresponding normalized Nambu wave functions are

$$
\begin{aligned}
|L, R\rangle & =\left[u_{\uparrow}(x), u_{\downarrow}(x), v_{\uparrow}(x), v_{\downarrow}(x)\right]^{T} \\
& =\frac{1}{\sqrt[4]{\pi \sigma^{2}}} e^{-\frac{\left(x-x_{0}\right)^{2}}{2 \sigma^{2}}} \chi_{L, R},
\end{aligned}
$$

with

$$
\chi_{L, R}=\left(\frac{-\sqrt{h+a}\left[\sqrt{h^{2}-a^{2}}+\operatorname{sgn}(\tau) i v\right]}{2 \sqrt{h}\left|\Delta_{0}\right|}, \frac{\sqrt{h-a}\left[i v+\operatorname{sgn}(\tau) \sqrt{h^{2}-a^{2}}\right]}{2 \sqrt{h}\left|\Delta_{0}\right|},-\operatorname{sgn}(\tau) \frac{\sqrt{h+a}}{2 \sqrt{h}} \frac{\Delta_{0}^{*}}{\left|\Delta_{0}\right|}, \frac{\sqrt{h-a}}{2 \sqrt{h}} \frac{\Delta_{0}^{*}}{\left|\Delta_{0}\right|}\right)^{T},
$$

where the left and right localized edge states $|L, R\rangle$ require $\operatorname{sgn}(\tau)=-1$ and 1 , respectively. One can check that the wave functions satisfy the symmetry requirement $\left|u_{\sigma}\right|=\left|v_{\sigma}\right|$ for Majorana modes. Finally, in order to compare with numerical results of our self-consistent BdG calculations, we define the symmetric and antisymmetric states $\left|\varphi^{ \pm}\right\rangle=(|L\rangle \pm|R\rangle) / \sqrt{2}$. We plot the analytical wave functions of the Majorana modes in Fig. 1(d), together with the numerical results. The agreement is remarkable.

In deriving the above results, we have assumed that $v<\left|\Delta_{0}\right|$. This is reasonable as for large $v$ the order parameter becomes vanishingly small. In the limit $v \ll\left|\Delta_{0}\right|$, the wave function of the Majorana mode takes the form

$$
\chi_{L, R}=\left(\frac{-(a+h)}{2 \sqrt{h^{2}+a h}}, \mp \frac{(h-a)}{2 \sqrt{h^{2}-a h}}, \pm \frac{\Delta_{0}^{*}}{2 \sqrt{h^{2}-a h}}, \frac{\Delta_{0}^{*}}{2 \sqrt{h^{2}+a h}}\right)^{T} .
$$

\section{EXPANSION OF THE FF STATE}

The characteristic signature of the FF state is finitemomentum pairing. Here, we show how this feature manifests itself in the density profiles of the atomic cloud during time-of-flight expansion. Figure 3 shows the time evolution of the density profiles for both an interacting and a noninteracting gas. Initially, the density profiles of both spin states are symmetric about the trap center. As the cloud expands, the profiles become asymmetric when $v \neq 0$. Furthermore, the center of mass of each spin state moves in opposite directions regardless of whether or not the atoms interact. There is, however, an important difference between an interacting and a noninteracting cloud. As shown in Fig. 4, for a noninteracting cloud, the center-of-mass positon of the whole cloud, defined as

$$
x_{\mathrm{c} . \mathrm{m} .}=\frac{1}{N} \sum_{\sigma=\uparrow, \downarrow} \int x n_{\sigma}(x) d x
$$

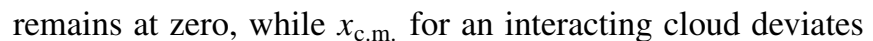
from zero as time increases. The deviation is stronger for larger interaction strength $\gamma$ and larger in-plane Zeeman field strength $|\nu|$. This result is consistent with a two-body calculation carried out by Dong et al. [19], where they found that the total mechanical momentum of the interaction-induced two-body bound state becomes finite as long as the in-plane Zeeman field is present. In the present study, the nonzero $x_{\mathrm{c} . \mathrm{m}}$. during the expansion is a direct consequence of the

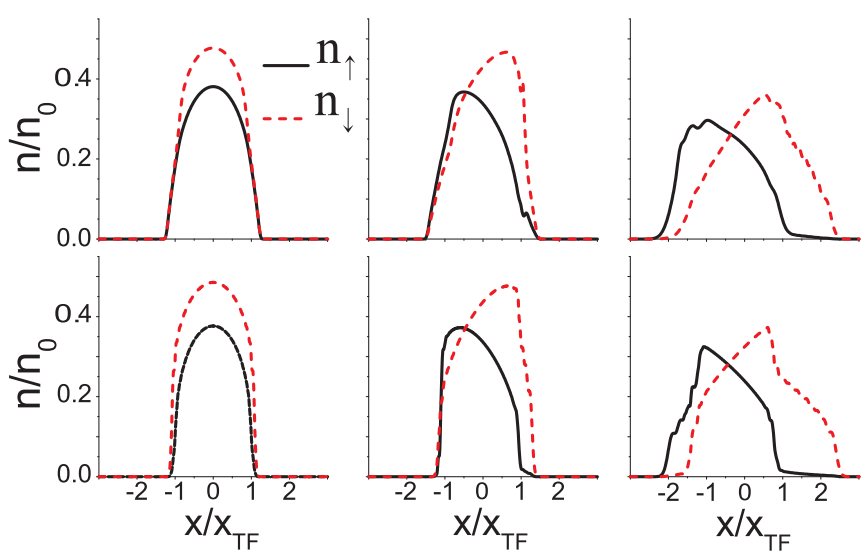

FIG. 3. (Color online) Expansion dynamics of the Fermi cloud released at time $t=0$. Top row: An interacting case with $\gamma=2.2$. Bottom row: A noninteracting case with $\gamma=0$. Other parameters are $\lambda=1.5 E_{F} / k_{F}, h=0.8 E_{F}$, and $v=0.2 E_{F}$. From left to right are plots of density profiles at $t=0,0.88 / \omega_{0}$, and $2.04 / \omega_{0}$, respectively. 


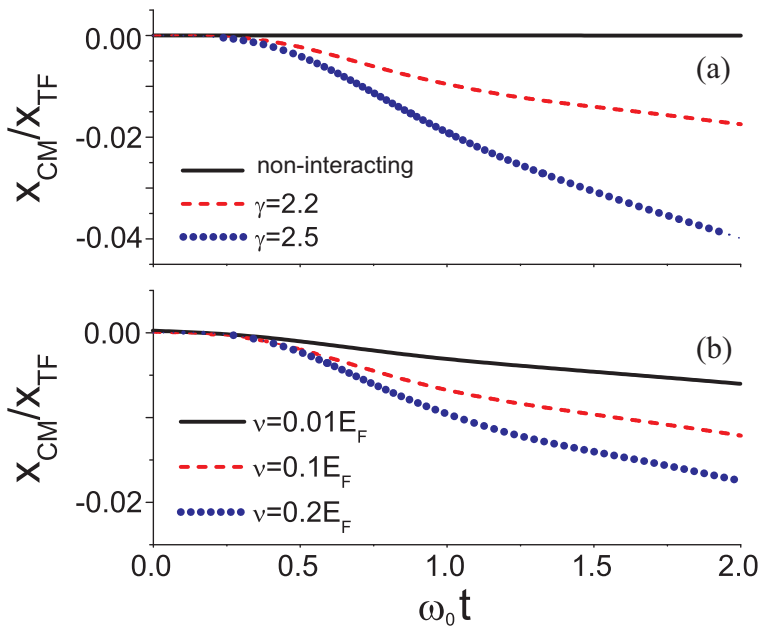

FIG. 4. (Color online) Center-of-mass position of the atomic cloud during free expansion. In (a), different curves represent different interaction strengths for $v=0.2 E_{F}$. In (b), different curves represent different in-plane Zeeman fields for $\gamma=2.2$. Other parameters are the same as in Fig. 3.

finite-momentum FF pairing in the original trapped system. That $x_{\mathrm{c} . \mathrm{m}}$. changes faster for larger interaction strength can be attributed to stronger pairing, and hence a larger fraction of the atoms form Cooper pairs with finite momentum.
Finally, we want to comment that the FF pairing here arises from an explicit breaking of the symmetry due to the presence of the in-plane Zeeman field. The sign of $v$ determines the sign of the pairing momentum. In other words, if we change the sign of $v, x_{\mathrm{c} . \mathrm{m}}$. will also change sign as a consequence.

\section{CONCLUSION}

We have considered both the static and dynamical properties of a trapped 1D Fermi gas subject to equal-weight Rashba-Dresselhaus spin-orbit coupling. This system enters an exotic topological FF state regime when a large effective Zeeman field with a nonzero in-plane component is present. The two salient features of this phase are (1) the presence of zero-energy Majorana modes localized near the edge of the trap and (2) finite-momentum pairing. These features manifest themselves in the density of states and the center-of-mass displacement during expansion, respectively. We hope future experiments may be able to realize and probe this interesting quantum phase.

\section{ACKNOWLEDGMENTS}

L.J. and E.T. acknowledge support from the US Army Research Office under Contract No. 60661PH. X.J.L. and H.H. are supported by the ARC Discovery Projects (DP140100637, FT 130100815, and DP140103231). H.P. is supported by the NSF and the Welch Foundation (Grant No. C-1669). We would like to thank Leslie Baksmaty for useful discussions.
[1] J. D. Sau, R. M. Lutchyn, S. Tewari, and S. Das Sarma, Phys. Rev. Lett. 104, 040502 (2010).

[2] Y. Oreg, G. Refael, and F. von Oppen, Phys. Rev. Lett. 105, 177002 (2010).

[3] V. Mourik, K. Zuo, S. M. Frolov, S. R. Plissard, E. P. A. M. Bakkers, and L. P. Kouwenhoven, Science 336, 1003 (2012).

[4] Y.-J. Lin, K. Jiménez-García, and I. B. Spielman, Nature (London) 471, 83 (2011).

[5] M. Sato, Y. Takahashi, and S. Fujimoto, Phys. Rev. Lett. 103, 020401 (2009).

[6] J. P. Vyasanakere, S. Zhang, and V. B. Shenoy, Phys. Rev. B 84, 014512 (2011).

[7] H. Hu, L. Jiang, X.-J. Liu, and H. Pu, Phys. Rev. Lett. 107, 195304 (2011).

[8] Z.-Q. Yu and H. Zhai, Phys. Rev. Lett. 107, 195305 (2011).

[9] M. Gong, S. Tewari, and C. Zhang, Phys. Rev. Lett. 107, 195303 (2011).

[10] W. Yi and G.-C. Guo, Phys. Rev. A 84, 031608(R) (2011).

[11] M. Iskin and A. L. Subaş1, Phys. Rev. Lett. 107, 050402 (2011).

[12] L. Han and C. A. R. Sá de Melo, Phys. Rev. A 85, 011606(R) (2012).

[13] P. Wang, Z.-Q. Yu, Z. Fu, J. Miao, L. Huang, S. Chai, H. Zhai, and J. Zhang, Phys. Rev. Lett. 109, 095301 (2012).

[14] L. W. Cheuk, A. T. Sommer, Z. Hadzibabic, T. Yefsah, W. S. Bakr, and M. W. Zwierlein, Phys. Rev. Lett. 109, 095302 (2012).

[15] X.-J. Liu and H. Hu, Phys. Rev. A 85, 033622 (2012).
[16] X.-J. Liu and P. D. Drummond, Phys. Rev. A 86, 035602 (2012).

[17] R. Wei and E. J. Mueller, Phys. Rev. A 86, 063604 (2012).

[18] T. Mizushima and M. Sato, New J. Phys. 15, 075010 (2013).

[19] L. Dong, L. Jiang, H. Hu, and H. Pu, Phys. Rev. A 87, 043616 (2013).

[20] L. Dong, L. Jiang, and H. Pu, New J. Phys. 15, 075014 (2013).

[21] V. B. Shenoy, Phys. Rev. A 88, 033609 (2013).

[22] Z. Zheng, M. Gong, X. Zou, C. Zhang, and G.-C. Guo, Phys. Rev. A 87, 031602(R) (2013).

[23] F. Wu, G.-C. Guo, W. Zhang, and W. Yi, Phys. Rev. Lett. 110, 110401 (2013).

[24] X.-J. Liu and H. Hu, Phys. Rev. A 87, 051608(R) (2013).

[25] X.-J. Liu and H. Hu, New J. Phys. 15, 093037 (2013).

[26] C. Qu, Z. Zheng, M. Gong, Y. Xu, L. Mao, X. Zou, G. Guo, and C. Zhang, Nat. Commun. 4, 2710 (2013).

[27] W. Zhang and W. Yi, Nat. Commun. 4, 2711 (2013).

[28] X.-J. Liu and H. Hu, Phys. Rev. A 88, 023622 (2013).

[29] C. Chen, Phys. Rev. Lett. 111, 235302 (2013).

[30] M. W. Zwierlein, A. Schirotzek, C. H. Schunck, and W. Ketterle, Science 311, 492 (2006).

[31] G. B. Partridge, W. Li, R. I. Kamar, Y.-A. Liao, and R. G. Hulet, Science 311, 503 (2006).

[32] S. Nascimbene, N. Navon, K. J. Jiang, L. Tarruell, M. Teichmann, J. McKeever, F. Chevy, and C. Salomon, Phys. Rev. Lett. 103, 170402 (2009). 
[33] Y.-A. Liao, A. S. C. Rittner, T. Paprotta, W. Li, G. B. Partridge, R. G. Hulet, S. K. Baur, and E. J. Mueller, Nature (London) 467, 567 (2010).

[34] B. Sundar and E. J. Mueller, Phys. Rev. A 88, 063632 (2013).

[35] X.-J. Liu, H. Hu, and P. D. Drummond, Phys. Rev. A 76, 043605 (2007).

[36] D. T. Colbert and W. H. Miller, J. Chem. Phys. 96, 1982 (1992).

[37] X.-J. Liu, Phys. Rev. A 87, 013622 (2013).

[38] K. Sun and C. J. Bolech, Phys. Rev. A 85, 051607(R) (2012).

[39] K. Sun and C. J. Bolech, Phys. Rev. A 87, 053622 (2013).

[40] G. Tonini, F. Werner, and Y. Castin, Eur. Phys. J. D 39, 283 (2006).
[41] K. J. Challis, R. J. Ballagh, and C. W. Gardiner, Phys. Rev. Lett. 98, 093002 (2007).

[42] R. G. Scott, F. Dalfovo, L. P. Pitaevskii, and S. Stringari, Phys. Rev. Lett. 106, 185301 (2011).

[43] H. Lu, L. O. Baksmaty, C. J. Bolech, and H. Pu, Phys. Rev. Lett. 108, 225302 (2012).

[44] P. Fulde and R. A. Ferrell, Phys. Rev. 135, A550 (1964).

[45] A. I. Larkin and Y. N. Ovchinnikov, Zh. Eksp. Teor. Fiz. 47, 1136 (1964) [Sov. Phys. JETP 20, 762 (1965)].

[46] L. Jiang, L. O. Baksmaty, H. Hu, Y. Chen, and H. Pu, Phys. Rev. A 83, 061604(R) (2011) 